\title{
What Does Imaging Reveal About the Pathology of Amyotrophic Lateral Sclerosis?
}

\author{
Martin R. Turner ${ }^{1,3}$ Esther Verstraete ${ }^{2,4}$ \\ Published online: 26 May 2015 \\ (C) The Author(s) 2015. This article is published with open access at Springerlink.com
}

\begin{abstract}
Amyotrophic lateral sclerosis (ALS) is now recognised to be a heterogeneous neurodegenerative syndrome of the motor system and its frontotemporal cortical connections. The development and application of structural and functional imaging over the last three decades, in particular magnetic resonance imaging (MRI), has allowed traditional post mortem histopathological and emerging molecular findings in ALS to be placed in a clinical context. Cerebral grey and white matter structural MRI changes are increasingly being understood in terms of brain connectivity, providing insights into the advancing degenerative process and producing candidate biomarkers. Such markers may refine the prognostic stratification of patients and the diagnostic pathway, as well as providing an objective assessment of changes in disease activity in response to future therapeutic agents. Studies are being extended to the spinal cord, and the application of neuroimaging to unaffected carriers of highly penetrant genetic mutations linked to the development of ALS offers a unique window to the pre-symptomatic landscape.
\end{abstract}

This article is part of the Topical Collection on Neuroimaging

Martin R. Turner

martin.turner@ndcn.ox.ac.uk

Esther Verstraete

E.Verstraete@umcutrecht.nl

1 Nuffield Department of Clinical Neurosciences, University of Oxford, Oxford, UK

2 University of Utrecht, Utrecht, Netherlands

3 John Radcliffe Hospital, West Wing Level 3, Oxford OX3 9DU, UK

4 University Medical Center, Heidelberglaan 100, Utrecht, Netherlands
Keywords Motor neurone disease - Magnetic resonance imaging · Positron emission tomography · Diffusion tensor imaging $\cdot$ Functional imaging $\cdot$ Cortical thickness

\section{Introduction}

Amyotrophic lateral sclerosis (ALS; also known as motor neuron disease or Lou Gehrig's disease) is an adult-onset neurodegenerative disorder of the motor system and its wider connections, characterized pathologically by loss of upper motor neurons of the primary motor cortex and corticospinal tract (CST) projections, together with lower motor neuron loss in the spinal anterior horns and pontomedullary nuclei [1]. Cytoplasmic inclusions of ubiquitinated TDP-43 are the molecular hallmark of $98 \%$ of cases, with a clinical syndrome unified by progressive and irreversible muscle denervation, and death typically arising from neuromuscular respiratory insufficiency. It appears that there are multiple and diverse upstream molecular routes to a final common clinical syndrome [2•].

There is no highly effective disease-modifying treatment for ALS. Despite a median survival from symptom onset of 3-4 years, the syndrome of ALS is surprisingly heterogeneous. Symptomatic onset appears to be focal, typically affecting either the distal limb or bulbar territories, with much rarer respiratory presentation. Subsequent spread of symptoms is not random, but appears to be defined by neuroanatomy with both contiguous and network propagation of pathology $[3,4 \bullet]$. There is a striking variability in the clinical burden of upper motor neuron (UMN) and lower motor neuron (LMN) signs. Extremes of LMN involvement have been historically termed as progressive muscular atrophy (PMA) and, a very rare UMN-only condition, primary lateral sclerosis (PLS). Both variants are associated with slower rates of 
progression but are considered part of the spectrum of ALS, which may be encompassed by the broader term, motor neuron disease.

Charcot's pivotal observation was the additional "deuteropathic" involvement of the CST in ALS, marking it out from the normally isolated "protopathic" LMN involvement of other peripheral neuromuscular disorders. He did not consider pathology above the brainstem, and it is striking that macroscopically, the primary motor cortex in ALS may appear unremarkable despite severe motor disability. Dedicated post mortem studies of the brain were rare until many decades later when motor pathway involvement was clear [5], even in those without clinical UMN signs [6]. Post mortem neuropathological research has suggested cerebral histopathological 'stages' in ALS depending on the extent of TDP-43 pathology [7•].

Although developments in neuropsychology [8,9], molecular histopathology [10] and genetics $[11,12]$ have confirmed beyond doubt that ALS lies on a spectrum with frontotemporal dementia (FTD), in fact, the clinical evidence was in the observations of the earliest pioneers of clinical neurology [13]. Overt dementia affects up to $15 \%$ of ALS patients, and is typically an early feature, associated with more rapid physical progression (termed ALS-FTD), though more subtle executive and behavioural dysfunction affects a much larger group of those with ALS, and is also an adverse prognostic sign [14]. ALS is recognised as a multisystem disorder and the link between classically motor and frontal lobe structures can be understood in the context of human evolution [15].

The ability to study the structure and function of the brain in vivo has been a major driver of the rapid advances in clinical neuroscience. ALS superficially appears dominated by its peripheral, more visible clinical manifestations, namely muscle wasting. Electromyography (EMG) has limited sensitivity for detecting sub-clinical denervation caused by loss of LMNs, but remains the current gold standard [16]. The reformulation of ALS as a fundamentally central nervous system disease pathologically has been underpinned by the application of neuroimaging to the brain, and more recently, also the spinal cord. Findings from neuroimaging have so far typically reflected the insights from post mortem neuropathological study, but also provided new insights through longitudinal studies and network analyses. It is essential to be aware of the limitations of these two techniques, which may nonetheless be regarded as complimentary in furthering our understanding of the pathogenesis in ALS (Table 1).

A coincident emerging research priority of establishing biomarkers in ALS applicable to therapeutic trials has independently brought neuroimaging to the forefront [17•]. ALS remains a fundamentally clinical diagnosis, and one in which delays of 12 months are commonplace. Diagnostic biomarkers, particularly for occult UMN involvement, would have clear value, but more so, biomarkers capable of stratifying patients for therapeutic trials, where heterogeneity may have been an important factor in the failure of drug studies to date. In addition, objective markers of disease activity and progression, rather than the current reliance on rate of change of functional rating scores (e.g., the revised ALS Functional Rating Score, ALSFRS) and survival as outcome measures, would permit faster 'no-go' decisions in therapeutic studies [18].

\section{The Dawn of Functional Neuroimaging: SPECT and PET}

In retrospect, the early observations using single-photon emission computed tomography (SPECT) of reduced frontotemporal binding in ALS patients with dementia [19], are entirely predictable with the modern recognition of an ALS-FTD spectrum. The earliest positron emission tomography (PET) studies of cerebral glucose metabolism noted widespread reductions, most obvious not only in those with a high burden of UMN clinical signs [20], but also in relation to more subtle neuropsychological deficits [21, 22]. PET brain activation studies in ALS began to consider the widened 'boundary

Table 1 Key characteristics of histopathological assessment versus neuroimaging

\begin{tabular}{|c|c|c|}
\hline & Histopathology & Neuroimaging \\
\hline Setting & Post mortem & In vivo \\
\hline Scale & Micro: single neurons and synapses & Macro: brain regions and pathways \\
\hline Scope & Sampled areas & Whole-brain, unbiased \\
\hline Quantification & By examiner & Semi-automated processing \\
\hline Timing & $\begin{array}{l}\text { Always cross-sectional, typically end-stage disease and } \\
\text { with potential for confounding by agonal state }\end{array}$ & $\begin{array}{l}\text { Cross-sectional or longitudinal in symptomatic cases, } \\
\text { and in familial cases also pre-symptomatic }\end{array}$ \\
\hline Specific potential & $\begin{array}{l}\text { Assessment of: } \\
\text { - Cell loss (type, layer, quantification) } \\
\text { - Protein inclusions } \\
\text { - Molecular characterization }\end{array}$ & $\begin{array}{l}\text { Assessment of: } \\
\text { - Functional reorganisation at the systems-level } \\
\text { - Network properties and efficiency }\end{array}$ \\
\hline
\end{tabular}


shift' of cerebral activation in relation to motor tasks [23] (Fig. 1). Aside from a clear indication of pathological effects beyond the primary motor cortex, this opened a wider debate that persists today, over the contribution of a disease-related wider recruitment of surviving neurons (perhaps involving plasticity mechanisms) versus a loss of local containment, e.g. through an inhibitory interneuronopathy. Finally, there has been renewed interest in the value of regional patterns of altered fluorodeoxyglucose (FDG) PET as part of the diagnostic pathway in ALS [24, 25].

\section{Ligand PET Studies}

The unrivalled sensitivity of PET has been exploited to understand aspects of the molecular pathology of ALS using radioligands, specifically neuroinflammation, inhibitory cortical influences and serotonin receptor changes (Fig. 1).

\section{Neuroinflammation}

The involvement of microglia and other neuroinflammatory processes in all neurodegenerative disorders is dogged by the issue of primary (pathogenic) versus secondary (reactive, possibly modulatory) involvement. It is clear that the situation is nuanced, possibly with a switch in core microglial function in early versus established disease phases [26, 27].
Until the development of PET ligands specific to activated microglia, evidence for neuroinflammation was largely derived from post mortem histopathology [28, 29]. In vivo studies with the first generation ligand PK11195 revealed evidence for activated microglia in both motor and frontal lobe regions [30]. Furthermore, the signal in both thalamic and primary motor cortex was strongly related to clinical UMN burden. Both findings were confirmed by a more recent study using a ligand with greater specificity [31]. Application of this ligand to rare unilateral forms of ALS (clinically termed Mills' syndrome) have confirmed topographically localized microglial activation [32], also demonstrated histopathologically [33].

\section{Cortical Inhibitory Function}

Paired stimulation studies using transcranial magnetic stimulation (TMS) have revealed the cortex to be hyperexcitable in ALS [34, 35]. This appears to be quite specific [36], and may predate the onset of symptoms [37]. This supports excitotoxic theories of ALS, which is also compatible with loss of inhibitory GABA-ergic interneuronal influences [38]. PET studies with the GABA-A receptor radioligand flumazenil demonstrated widespread reductions in binding [39], consistent with a loss of inhibitory interneurons, and this was confirmed histopathologically [40, 41]. Moreover, a study comparing sporadic ALS cases with a uniformly more slowly progressive

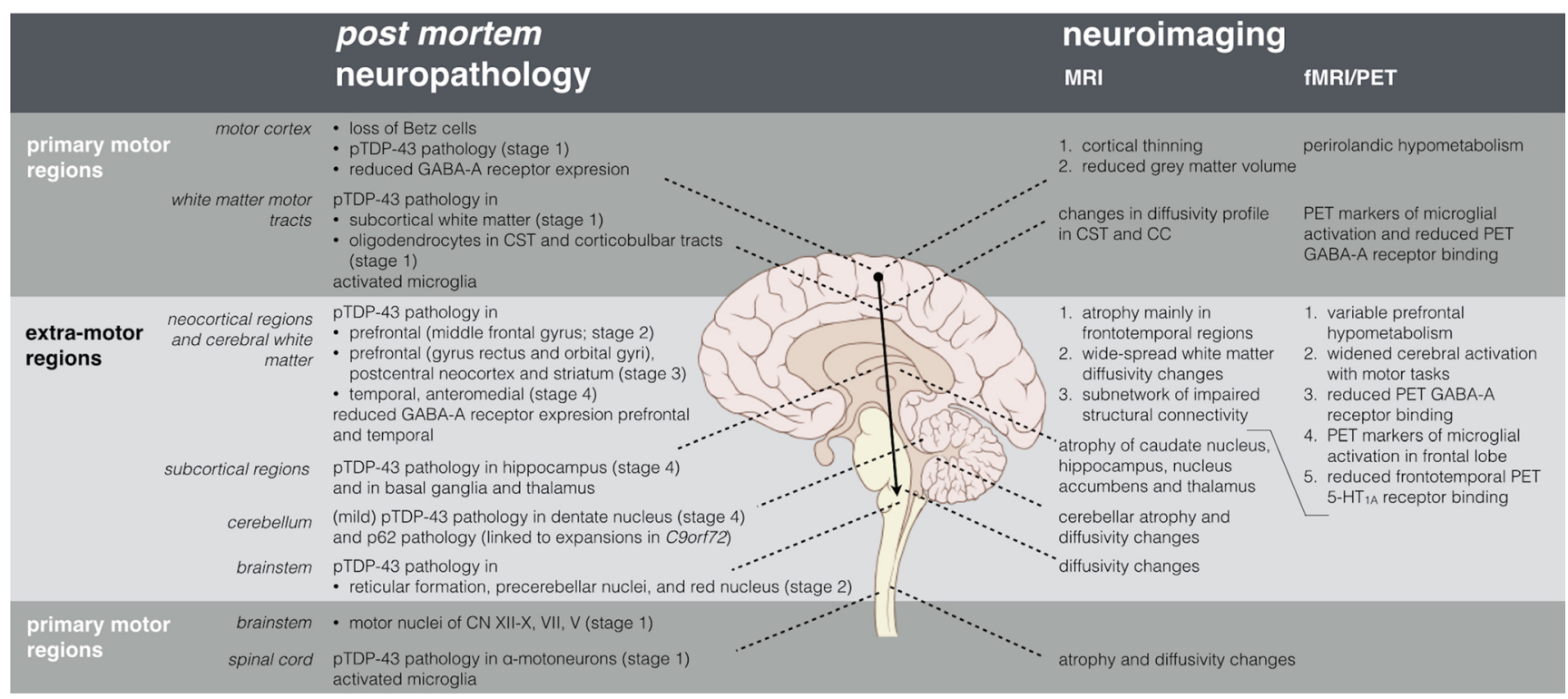

Fig. 1 Summary of the pathogenic insights from post mortem histopathological and neuroimaging research in ALS. The findings in the primary motor regions are separately shown from the findings in extra-motor regions. The primary motor regions are defined as the motor cortex (precentral gyrus and paracentral lobule or Brodmann area 4 and 6), the major white matter tracts (corticospinal tracts [CST] and corpus callosum $[\mathrm{CC}]$ ), the motor nuclei in the brainstem and motoneurons arising from the spinal cord anterior horns. The extra- motor regions are here defined as all other brain regions. TDP-43 pathology is defined according to the post mortem histological stages proposed by Brettschnieder and colleagues [7•]. pTDP-43 phosphorylated, 43-kDa TAR DNA-binding protein, CST corticospinal tracts, $C C$ corpus callosum, $C N$ cranial nerves, $P E T$ positron emission tomography, GABA-A receptor gamma-aminobutyric acid receptor type A, 5-HT1A receptor 5-hydroxytryptamine receptor type $1 \mathrm{~A}$ 
familial form of the condition (matched for clinical features other than disease duration), showed preservation of binding in motor regions of the latter [42], supporting a role for this cell type in pathogenesis.

\section{Serotonergic Function}

PET studies in both ALS [43], and later FTD [44], using a ligand with affinity for the serotonin $\left(5-\mathrm{HT}_{1 \mathrm{~A}}\right)$ receptor, showed marked frontotemporal reductions in binding, confirmed histopathologically in FTD patients [45]. This receptor may be a marker of pyramidal cell loss, or have a more specific role in motor neuronal control or cognition with relevance to both ALS and FTD, and warrants further study.

\section{Clinical MRI}

The principle role of clinical magnetic resonance imaging (MRI) has been in the exclusion of structural pathology mimicking clinical UMN and LMN involvement, principally in the spinal cord [46]. The ubiquity and non-invasive nature of MRI has supported its continued development and a leading role in ALS biomarker discovery [47]. The first application of MRI to a small group of ALS patients highlighted corticospinal tract hyperintensity, particularly visible on T2-weighted sequences [48]. It is neither sensitive nor specific for ALS, though similar changes seen in hepatic failure may provide a clue to common mechanisms of oxidative stress and selective vulnerability [49].

\section{Advanced Structural MRI}

The development of automated segmentation and analysis tools (voxel- and surface-based morphometry), and new acquisition sequences focused on the directionality of the movement of water (diffusion tensor imaging, DTI), have allowed unprecedented in vivo neuropathological study of the brain in ALS (Fig. 1).

\section{Grey Matter}

One of the first automated, quantitative, post-processing imaging tools was voxel-based morphometry (VBM), allowing group comparisons of grey and white matter density profiles to provide an indication of regional brain atrophy patterns. The initial VBM studies in ALS showed diverging results, ranging from focal atrophy in motor regions (precentral gyrus) [50], to widespread atrophy in mostly frontal and temporal regions sparing involvement of the motor cortex [51]. These variable results seem to have been caused by differences in image processing algorithms and statistical models [52].
With increasing patient numbers, however, subgroup analyses comparing bulbar- versus limb-onset patients have shown different atrophy patterns within the motor strip in correspondence with functional disabilities $[53 \bullet, 54 \bullet]$. These results are suggestive of focal degenerative cortical changes. Also, the comparison between cognitively impaired and unimpaired patients has demonstrated different patterns of atrophy in which impaired patients show more widespread atrophy extending to extra-motor regions, defined as anatomical regions outside the primary motor regions, mainly in the frontotemporal lobes $[55,56]$.

Besides VBM, another technique to quantify grey and white matter volumes has emerged, based on the reconstruction of the boundaries between grey and white matter. This technique is referred to as surface-based morphometry (SBM), or cortical thickness measurement and has been successfully applied in ALS. Where VBM is typically based on group comparisons, SBM can provide individual morphometry measures and more detailed information on potential shape alterations. SBM studies have consistently demonstrated cortical thinning in the precentral gyrus or primary motor regions in patients with ALS $[57,58]$. Studies comparing volume, thickness and surface area of the cortical mantle have repeatedly shown that cortical thickness has the greatest sensitivity to the disease-related changes in ALS [56, 57]. In addition, similar to what was demonstrated in VBM studies, focal thinning of the motor cortex mirrors clinical features of ALS [58, 59॰].

Extra-motor involvement has also been shown more pronounced in patients with additional cognitive deficits and along the course of disease in ALS [60]. This extra-motor cortical thinning is probably the imaging correlate of the widespread degenerative changes, which have also been demonstrated in postmortem studies [61]. It may be important to quantify the extent of UMN and extra-motor involvement since the extent of neuronal involvement correlates with survival, with ALS-FTD typically showing a worse prognosis compared to isolated lower or upper motor neuron phenotypes such as PMA and PLS [62].

In addition to cortical grey matter, recent structural imaging studies have focused specifically on the sub-cortical structures in ALS using VBM- or SBM-based analyses. The caudate nucleus, hippocampus and nucleus accumbens were particularly notable as showing progressive atrophy over time [63, 64], and also the thalamus [54•], in keeping with the earlier observation of intense microglial infiltration of this structure [30]. Such observations are in line with post mortem studies $[61,65]$, so that neuroimaging now holds the unique promise to reveal the sequence of events along the course of disease, and not just the end stage.

Cerebellar involvement in ALS has gained increased recognition in the past years [66], and structural imaging has revealed unique cerebellar atrophy patterns in ALS, ALS- 
FTD and FTD, in relation to neuropsychiatric and motor characteristics [67]. This is in alignment with neuropathological findings showing ubiquitin-positive inclusions in the cerebellum, particularly in the granular layer in ALS and FTD [68]. The cerebellum, as a relay station that includes multiple reciprocal cortico-cerebellar connections, appears not to escape disease-related degenerative changes in ALS (akin to the thalamus), despite the lack of overt clinical manifestations.

\section{White Matter}

The most extensively applied technique to study the white matter changes in the ALS brain is diffusion tensor imaging (DTI), which makes use of the typical microstructural organization of white matter as parallel oriented fibres. By assessing the diffusivity characteristics of brain tissue, it becomes possible to identify white matter and assess its integrity. Intact white matter will restrict diffusion of water parallel to the fibre direction, while damaged white matter will cause less restricted diffusivity (quantifiable using measures such as reduced fractional anisotropy, FA or increased mean diffusivity, $\mathrm{MD}$ ). Based on the main diffusion direction, it is possible to track the course of white matter tracts, which is referred to as tractography. Tractography allows reconstruction of the white matter tracts and thus the brains structural connectivity [69].

It is recognised that the CSTs and corpus callosum (CC) in ALS, as the main white matter motor tracts, have altered diffusivity characteristics compared to healthy controls [17•]. The FA is reduced with an increase in MD, and the more sensitive measure of radial diffusivity (RD) $[70,71]$. This altered diffusivity profile might be simply due to loss of white matter fibres as a result of the neurodegenerative process, but may also be compatible with the microglial activation seen in vivo using PET $[30,31]$ and post mortem histopathology [72]. Currently, there are no systematic studies comparing diffusivity changes in the ALS brain with histopathological white matter changes but this is an active area of research in order to understand better the tissue correlates for DTI changes [73], including in relation to iron deposition [74]. Despite the fact that DTI studies have consistently demonstrated the involvement of the CST at a group level, its diagnostic accuracy for individual ALS patients is currently insufficient [75]. The heterogeneity of CST involvement in cases of ALS without clinical UMN involvement has also been demonstrated in neuropathological studies [76].

In line with the widespread grey matter atrophy, white matter changes have also been shown to extend beyond the primary motor tracts in ALS. Voxel-based DTI studies demonstrated this and, in parallel to patterns of grey matter atrophy, these changes were extensive [77], particularly in patients with cognitive impairment $[55,78]$. A large longitudinal study suggested that MRI is sensitive to extensive grey matter changes in ALS [54•].

More recent tract-based analyses, albeit at group rather than individual level, support the view that these widespread white matter changes make up a sub-network of impaired connectivity, typically involving primary and secondary motor connections [79, 80]. Over time, this sub-network of impaired connectivity has been shown to expand, suggesting a network basis for spread of pathology [4•]. Neuropathological studies have shown that phosphorylated TDP-43 can be more or less distributed in the brain, defined as histopathological (rather than clinical) stages [7•]. It does not appear however that every ALS patient will eventually proceed to develop all histopathological stages, and there is particular evidence for the influence of genotype in this respect. For example, it has been shown that ALS patients associated with hexanucleotide repeat expansions in C9orf72 are more prone to develop ALS with cognitive impairment, which is also related to more extensive cerebral histopathological change [81]. On the contrary, ALS linked to mutations superoxide dismutase (SOD1) [82], or the more recently discovered $\mathrm{CHCH} 10 \mathrm{mu}-$ tations [83], typically develop a pure motor phenotype in which pathology stays confined to motor regions. Neuroimaging offers unique potential for exploration of in vivo pathological staging of disease [84•], that can be integrated with efforts at clinical staging [85].

\section{Functional MRI}

Blood oxygenation level-dependent (BOLD) MRI has been a valuable tool for the study of brain function. Functional MRI (fMRI) acquisition during motor and cognitive tasks in ALS has now developed into studying the coherence of regional signals under resting-state conditions [86], which is particular suited for a physically disabling condition such as ALS where it is challenging to standardize motor responses during taskbased MRI.

\section{Task-Based fMRI}

In line with results from activation PET [23], task-based fMRI studies in ALS have demonstrated increased cortical activation associated with motor processing [87, 88]. Taskbased fMRI studies can provide unique insights into processes like functional reorganization, which are most easily explained as recruitment of surviving neurons in an attempt to compensate for structural loss. Besides motor tasks, the functional response to cognitive tasks has also been studied in ALS, including using anti-saccade task of executive dysfunction [89]. A notable recent study reported prefrontal activation abnormalities related to letter fluency in patients with clinically LMN-only form of ALS [90], providing 
further evidence for a continuum of cerebral involvement across the range of the ALS syndrome, independent of clinical UMN involvement.

\section{Task-Free (Resting-State) fMRI}

Resting-state functional MRI (rs-fMRI) uses the basal brain activity to define functional connectivity between brain regions based on the degree of synchronization of low frequency oscillations $[91,92]$. Both regional, as well as whole-brain approaches have been used to study functional connectivity in ALS. Resting-state networks were first investigated in ALS patients using independent component analysis [93]. Of the five resting-state networks extracted, ALS patients showed reduced functional connectivity in the default mode and sensorimotor networks in ALS. The default mode network comprises medial frontal regions, parietal regions, inferior temporal gyrus, cingulate cortex and precuneus, and is consistently deactivated during the performance of cognitive tasks [94, 95]. The sensorimotor network is composed of the primary motor cortex, anterior cingulate cortex, somatosensory regions and auditory cortex [96, 97].

Reduced functional connectivity in the motor network was replicated in other studies [98, 99]. However, subsequent studies have found increased functional activity within and beyond the motor and premotor cortex, despite reduced structural connectedness [100], or a combination of increased and decreased functional coherence within cortical sub-regions [101-103]. A recent study aiming to address the link between functional and structural connectivity in ALS assessed all affected structural connections in ALS and showed an overall positive correlation between structural and functional connectivity [104]. A paradoxical relation between structural and functional connectivity exists in specific sub-regional connections which may be related to compensatory mechanisms, but perhaps also drive pathogenesis more directly [100].

In line with previous PET but also structural imaging studies, rs-fMRI has shown connectivity changes in the default mode and frontoparietal networks in relation to cognition and behaviour in ALS. Enhanced parietal connectivity was associated with the clinical and cognitive deficits of the patients and suggested to be a compensatory mechanism [103]. In line with this study, a study comparing both FTD (behavioural variant) and ALS patient groups showed increased signal in the posterior cingulate cortex in FTD patients that were suppressed in ALS patients. Overall, however, functional connectivity patterns in ALS and FTD were found to be strikingly similar as may be expected in diseases belonging to the same clinicopathological spectrum [105]. It is hoped that techniques like magnetoencephalography (MEG), with a much higher temporal resolution compared to fMRI, will help to further unravel functional connectivity changes in ALS [106].

\section{Magnetic Resonance Spectroscopy}

The metabolite content of tissue can be explored using magnetic resonance spectroscopy (MRS). The most robustly distinguishable metabolites in the brain with proton MRS are Nacetyl aspartate (NAA, a marker of neuronal density), choline (Cho, a marker of membrane integrity) and creatine $(\mathrm{Cr}$, a marker of cellular energetics). NAA is typically expressed as a ratio with one or both of Cho and $\mathrm{Cr}$. In ALS, NAA reductions in the primary motor cortex have been among the most consistent findings, initially using single-voxel approaches [107], and more recently, whole-brain analysis [108]. Glutamate and glutamine increases in the brainstem have supported excitotoxic theories of ALS [109], with equally consistent reductions in GABA reported more recently [110].

\section{Advanced Spinal Cord MRI}

Supported by studies using SOD1 transgenic mouse models of ALS, which are necessarily biased towards the study of LMNs, the concept of ALS as a 'dying back' pathological process from the LMNs to involve the CSTs and wider brain has persisted. This is despite recognition of the overlap between ALS and FTD, and with cognitive involvement preceding that of the downstream motor system in a significant proportion of cases. The study of spinal cord pathology in vivo would have particular value in furthering knowledge about pathological spread and the variation in UMN and LMN involvement clinically. Issues with resolution, physiological movement and the close proximity of bone have made the application of MRI challenging [111]. Nonetheless, it has been possible to demonstrate structural changes in the cord in ALS, as well as show sub-clinical involvement of sensory pathways [112•].

\section{Genetic Imaging}

A diverse group of genetic mutations has been linked to a small proportion of cases with the syndrome of ALS [113]. Pathological mutations in SOD1 and hexanucleotide expansions in C9orf72 account for over half of the $5 \%$ of ALS cases reporting a clear family history of either ALS or FTD. Whereas cognitive or behavioural involvement is exceptional in SOD1mediated ALS, it is common in C9orf72 expansion-associated ALS where carriers within the same family may develop either ALS-FTD or pure FTD [114]. MRI studies in ALS patient carriers of $C 9$ orf 72 expansions revealed extensive changes, including the involvement of sub-cortical structures such as the thalami and clinically silent regions, e.g. cerebellum, despite attempts to control for the greater cognitive involvement in such patients compared to the sporadic ALS comparator group 
[115]. Similar more widespread changes in FDG uptake were noted in a PET study of C9orf72-related ALS patients [116]. DTI studies in patients homozygous for the 'D90A' SOD1 mutation associated with a consistently slower form of the disease, showed strikingly preserved CST integrity despite similar UMN burden clinically [70], which may reflect altered regional white matter vulnerability.

\section{Pre-symptomatic Studies}

Carriers of genetic mutations linked to the development of ALS offer a unique window to study the pre-symptomatic pathological landscape, both to understand the very earliest changes and to open the possibility of neuroprotection in the longer term [117]. A flumazenil PET study of two presymptomatic SOD1 mutation carriers hinted at left operculum region changes similar to those seen in affected patients [42]. DTI studies in other SOD1 patients have been conflicting, with one showing bilaterally reduced FA in the region in the internal capsule [118] but another no significant difference [119]. The metabolic profile of the cervical cord in a pre-symptomatic SOD1 patient study using MRS was more aligned to affected patients than healthy controls [120]. Combined DTI and rs-fMRI studies in pre-symptomatic carriers of FTD-only related genes (MAPT and $P R G N$ ) found evidence of both structural and functional change [121], with the expectation that there will be similar findings in C9orf 72 expansion carriers when the results of such studies are known.

It has also been possible to explore the pre-symptomatic period by applying MRI to the transgenic $S O D 1$ mouse model of ALS. This has revealed T2-weighted intensity changes in brainstem nuclei, corresponding to vacuolation and microglial infiltration histologically [122•], and which could be modified with anti-inflammatory therapy [123].

\section{Multimodal Analyses}

At present, the variation in brain structure and function has made the application of advanced MRI techniques limited to group-level differences. However, it is hoped that a combination of measures from different acquisitions can provide a signature applicable at the individual patient level. Support for this discriminatory improvement has come from combined studies of structural and functional MRI [100], and from combined DTI and MRS [124, 125]. It has also been possible to link levels of neurofilament light chain in the CSF of ALS patients to the structural integrity of the CSTs as measured by DTI [126], linking biochemical and neuroimaging biomarkers directly.

\section{Standardization and Harmonization}

In 2010, the Neuroimaging Society in ALS (www.NiSALS.org) was formed by a group of international scientists with a focus on the application of neuroimaging to the study of ALS. It has met annually to consider new developments in the field, but also to develop a standardized protocol for use as a source of outcome measures in therapeutic trials [127]. The establishment of a data repository has allowed the first multi-centre studies, which have confirmed the feasibility of MRI in this setting despite the undoubted major challenges of intra- and inter-site variability.

\section{Concluding Remarks}

Neuroimaging has brought unparalleled in vivo pathological insights into ALS, now also starting to include the pre-symptomatic landscape. The precedent set in Alzheimer's disease by the development of an in vivo radioligand for the intracellular molecule tau [128], opens the possibility of a TDP-43 equivalent that might have particular value in the pre-symptomatic period. In addition, core mechanisms may be revealed that have implications for future therapeutics in ALS and neuroimaging has shown its great potential for generating biomarkers that can be used to efficiently test them. Combining traditional neuropathology with the power of in vivo neuroimaging data offers the best opportunity to further understanding of pathogenesis in ALS.

\section{Compliance with Ethics Guidelines}

Conflict of Interest Martin R. Turner declares that he has no conflict of interest. Esther Verstraete has received consultancy fees from ALS Imaging Advisory Board and Biogen Idec.

Human and Animal Rights and Informed Consent This article does not contain any studies with human or animal subjects performed by any of the authors.

Open Access This article is distributed under the terms of the Creative Commons Attribution 4.0 International License (http:// creativecommons.org/licenses/by/4.0/), which permits unrestricted use, distribution, and reproduction in any medium, provided you give appropriate credit to the original author(s) and the source, provide a link to the Creative Commons license, and indicate if changes were made.

\section{References}

Papers of particular interest, published recently, have been highlighted as:

- Of importance

1. Kiernan MC, Vucic S, Cheah BC, Turner MR, Eisen A, Hardiman O, et al. Amyotrophic lateral sclerosis. Lancet. 2011;377:942-55. 
2. Turner MR, Swash M. The expanding syndrome of amyotrophic lateral sclerosis: a clinical and molecular odyssey. J Neurol Neurosurg Psychiatry. 2015. This review explores the increasing genetic and cellular biological complexity of ALS in relation to the core clinical syndrome described by the pioneers of clinical neurology and its overlap with FTD.

3. Ravits JM, La Spada AR. ALS motor phenotype heterogeneity, focality, and spread: deconstructing motor neuron degeneration. Neurology. 2009;73:805-11.

4. Verstraete E, Veldink JH, van den Berg LH, van den Heuvel MP. Structural brain network imaging shows expanding disconnection of the motor system in amyotrophic lateral sclerosis. Hum Brain Mapp. 2014;35:1351-61. Pioneering network-based analysis of structural MRI data at two time points, showing an expanding network of impaired sub-connections, and supporting an emerging concept of pathological spread occuring along neuronal connections.

5. Smith MC. Nerve fibre degeneration in the brain in amyotrophic lateral sclerosis. J Neurol Neurosurg Psychiatry. 1960;23:269-82.

6. Brownell B, Oppenheimer DR, Hughes JT. The central nervous system in motor neurone disease. J Neurol Neurosurg Psychiatry. 1970;33:338-57.

7. Brettschneider J, Del Tredici K, Toledo JB, Robinson JL, Irwin DJ, Grossman M, et al. Stages of pTDP-43 pathology in amyotrophic lateral sclerosis. Ann Neurol. 2013;74:20-38. A groundbreaking study of post mortem ALS brains, looking at the regional burden of the signature cellular pathology, which was categorised by its extent into discrete histopathological stages.

8. Gallassi R, Montagna P, Ciardulli C, Lorusso S, Mussuto V, Stracciari A. Cognitive impairment in motor neuron disease. Acta Neurol Scand. 1985;71:480-4.

9. Phukan J, Elamin M, Bede P, Jordan N, Gallagher L, Byrne S, et al. The syndrome of cognitive impairment in amyotrophic lateral sclerosis: a population-based study. J Neurol Neurosurg Psychiatry. 2012;83:102-8.

10. Neumann M, Sampathu DM, Kwong LK, Truax AC, Micsenyi $\mathrm{MC}$, Chou TT, et al. Ubiquitinated TDP-43 in frontotemporal lobar degeneration and amyotrophic lateral sclerosis. Science (New York, NY). 2006;314:130-3.

11. Renton AE, Majounie E, Waite A, Simon-Sanchez J, Rollinson S, Gibbs JR, et al. A hexanucleotide repeat expansion in C9ORF72 is the cause of chromosome 9p21-linked ALS-FTD. Neuron. 2011;72:257-68.

12. DeJesus-Hernandez M, Mackenzie IR, Boeve BF, Boxer AL, Baker M, Rutherford NJ, et al. Expanded GGGGCC hexanucleotide repeat in noncoding region of C9ORF72 causes chromosome 9p-linked FTD and ALS. Neuron. 2011;72:245-56.

13. Alzheimer A. Über einen Fall von spinaler progressiver Muskelatrophie mit hinzutretender Erkrankung bulbärer Kerne in der Rinde. Archiv Psychiatrie. 1891;23:459-85.

14. Elamin M, Phukan J, Bede P, Jordan N, Byrne S, Pender N, et al. Executive dysfunction is a negative prognostic indicator in patients with ALS without dementia. Neurology. 2011;76:1263-9.

15. Eisen A, Turner MR, Lemon R. Tools and talk: an evolutionary perspective on the functional deficits associated with amyotrophic lateral sclerosis. Muscle Nerve. 2014;49:469-77.

16. Douglass CP, Kandler RH, Shaw PJ, McDermott CJ. An evaluation of neurophysiological criteria used in the diagnosis of motor neuron disease. J Neurol Neurosurg Psychiatry. 2010;81:646-9.

17. Turner MR, Agosta F, Bede P, Govind V, Lule D, Verstraete E. Neuroimaging in amyotrophic lateral sclerosis. Biomark Med. 2012;6:319-37. A comprehensive review of all neuroimaging (SPECT, PET and MRI) in ALS, including online tables listing the main findings of every study.

18. Turner MR, Kiernan MC, Leigh PN, Talbot K. Biomarkers in amyotrophic lateral sclerosis. Lancet Neurol. 2009;8:94-109.
19. Neary D, Snowden JS, Mann DM, Northen B, Goulding PJ, Macdermott N. Frontal lobe dementia and motor neuron disease. J Neurol Neurosurg Psychiatry. 1990;53:23-32.

20. Dalakas MC, Hatazawa J, Brooks RA, Di Chiro G. Lowered cerebral glucose utilization in amyotrophic lateral sclerosis. Ann Neurol. 1987;22:580-6.

21. Ludolph AC, Langen KJ, Regard M, Herzog H, Kemper B, Kuwert T, et al. Frontal lobe function in amyotrophic lateral sclerosis: a neuropsychologic and positron emission tomography study. Acta Neurol Scand. 1992;85:81-9.

22. Kew JJ, Goldstein LH, Leigh PN, Abrahams S, Cosgrave N, Passingham RE, et al. The relationship between abnormalities of cognitive function and cerebral activation in amyotrophic lateral sclerosis. A neuropsychological and positron emission tomography study. Brain J Neurol. 1993;116(Pt 6):1399-423.

23. Kew JJ, Leigh PN, Playford ED, Passingham RE, Goldstein LH, Frackowiak RS, et al. Cortical function in amyotrophic lateral sclerosis. A positron emission tomography study. Brain J Neurol. 1993;116(Pt 3):655-80.

24. Pagani M, Chio A, Valentini MC, Oberg J, Nobili F, Calvo A, et al. Functional pattern of brain FDG-PET in amyotrophic lateral sclerosis. Neurology. 2014;83:1067-74.

25. Van Laere K, Vanhee A, Verschueren J, De Coster L, Driesen A, Dupont $\mathrm{P}$, et al. Value of 18 fluorodeoxyglucose-positron-emission tomography in amyotrophic lateral sclerosis: a prospective study. JAMA Neurol. 2014;71:553-61.

26. Evans MC, Couch Y, Sibson N, Turner MR. Inflammation and neurovascular changes in amyotrophic lateral sclerosis. Mol Cell Neurosci. 2012;53:34-41.

27. Murdock BJ, Bender DE, Segal BM, Feldman EL. The dual roles of immunity in ALS: injury overrides protection. Neurobiol Dis. 2015.

28. Troost D, Van den Oord JJ, Vianney de Jong JM. Immunohistochemical characterization of the inflammatory infiltrate in amyotrophic lateral sclerosis. Neuropathol Appl Neurobiol. 1990;16:401-10.

29. Troost D, Claessen N, Van den Oord JJ, Swaab DF, de Jong JM. Neuronophagia in the motor cortex in amyotrophic lateral sclerosis. Neuropathol Appl Neurobiol. 1993;19:390-7.

30. Turner MR, Cagnin A, Turkheimer FE, Miller CC, Shaw CE, Brooks DJ, et al. Evidence of widespread cerebral microglial activation in amyotrophic lateral sclerosis: an [(11)C](R)-PK11195 positron emission tomography study. Neurobiol Dis. 2004;15: 601-9.

31. Zurcher NR, Loggia ML, Lawson R, Chonde DB, IzquierdoGarcia D, Yasek JE, et al. Increased in vivo glial activation in patients with amyotrophic lateral sclerosis: Assessed with [(11)C]-PBR28. NeuroImage Clin. 2015;7:409-14.

32. Turner MR, Gerhard A, Al-Chalabi A, Shaw CE, Hughes RAC, Banati RB, et al. Mills' and other isolated upper motor neuron syndromes: in vivo study with [11C]-PK11195 PET. J Neurol Neurosurg Psychiatry. 2005;76:871-4.

33. Baumer D, Butterworth R, Menke RA, Talbot K, Hofer M, Turner MR. Progressive hemiparesis (Mills syndrome) with aphasia in amyotrophic lateral sclerosis. Neurology. 2014;82:457-8.

34. Yokota T, Yoshino A, Inaba A, Saito Y. Double cortical stimulation in amyotrophic lateral sclerosis. J Neurol Neurosurg Psychiatry. 1996;61:596-600.

35. Ziemann U, Winter M, Reimers CD, Reimers K, Tergau F, Paulus W. Impaired motor cortex inhibition in patients with amyotrophic lateral sclerosis. Evidence from paired transcranial magnetic stimulation. Neurology. 1997;49:1292-8.

36. Vucic S, Ziemann U, Eisen A, Hallett M, Kiernan MC. Transcranial magnetic stimulation and amyotrophic lateral sclerosis: pathophysiological insights. J Neurol Neurosurg Psychiatry. 2013;84:1161-70. 
37. Vucic S, Nicholson GA, Kiernan MC. Cortical hyperexcitability may precede the onset of familial amyotrophic lateral sclerosis. Brain J Neurol. 2008;131:1540-50.

38. Turner MR, Kiernan MC. Does interneuronal dysfunction contribute to neurodegeneration in amyotrophic lateral sclerosis? Amyotroph Lateral Scler Off Publ World Fed Neurol Res Group Motor Neuron Dis. 2012;13:245-50.

39. Lloyd CM, Richardson MP, Brooks DJ, Al Chalabi A, Leigh PN. Extramotor involvement in ALS: PET studies with the GABA(A) ligand [(11)C]flumazenil. Brain J Neurol. 2000;123(Pt 11):228996.

40. Maekawa S, Al-Sarraj S, Kibble M, Landau S, Parnavelas J, Cotter $\mathrm{D}$, et al. Cortical selective vulnerability in motor neuron disease: a morphometric study. Brain J Neurol. 2004;127:1237-51.

41. Petri S, Kollewe K, Grothe C, Hori A, Dengler R, Bufler J, et al. GABA(A)-receptor mRNA expression in the prefrontal and temporal cortex of ALS patients. J Neurol Sci. 2006;250:124-32.

42. Turner MR, Hammers A, Al-Chalabi A, Shaw CE, Andersen PM, Brooks DJ, et al. Distinct cerebral lesions in sporadic and 'D90A' SOD1 ALS: studies with [11C]flumazenil PET. Brain J Neurol. 2005;128:1323-9.

43. Turner MR, Rabiner EA, Hammers A, Al-Chalabi A, Grasby PM, Shaw CE, et al. [11C]-WAY100635 PET demonstrates marked 5HT1A receptor changes in sporadic ALS. Brain J Neurol. 2005;128:896-905

44. Lanctot KL, Herrmann N, Ganjavi H, Black SE, Rusjan PM, Houle S, et al. Serotonin-1A receptors in frontotemporal dementia compared with controls. Psychiatry Res. 2007;156:247-50.

45. Bowen DM, Procter AW, Mann DM, Snowden JS, Esiri MM, Neary D, et al. Imbalance of a serotonergic system in frontotemporal dementia: implication for pharmacotherapy. Psychopharmacology (Berl). 2008;196:603-10.

46. Filippi M, Agosta F, Abrahams S, Fazekas F, Grosskreutz J, Kalra $\mathrm{S}$, et al. EFNS guidelines on the use of neuroimaging in the management of motor neuron diseases. Eur J Neurol Off J Eur Fed Neurol Soc. 2010;17:526-33.

47. Turner MR. MRI as a frontrunner in the search for amyotrophic lateral sclerosis biomarkers? Biomark Med. 2011;5:79-81.

48. Goodin DS, Rowley HA, Olney RK. Magnetic resonance imaging in amyotrophic lateral sclerosis. Ann Neurol. 1988;23:418-20.

49. Turner MR. Reversible diffusion MRI abnormalities and transient mutism after liver transplantation. Neurology. 2005;64:177. author reply.

50. Agosta F, Pagani E, Rocca MA, Caputo D, Perini M, Salvi F, et al. Voxel-based morphometry study of brain volumetry and diffusivity in amyotrophic lateral sclerosis patients with mild disability. Hum Brain Mapp. 2007;28:1430-8.

51. Mezzapesa DM, Ceccarelli A, Dicuonzo F, Carella A, De Caro MF, Lopez M, et al. Whole-brain and regional brain atrophy in amyotrophic lateral sclerosis. AJNR Am J Neuroradiol. 2007;28: 255-9.

52. Rajagopalan V, Yue GH, Pioro EP. Do preprocessing algorithms and statistical models influence voxel-based morphometry (VBM) results in amyotrophic lateral sclerosis patients? A systematic comparison of popular VBM analytical methods. J Magn Reson Imaging. 2014;40:662-7.

53. Bede P, Bokde A, Elamin M, Byrne S, McLaughlin RL, Jordan N, et al. Grey matter correlates of clinical variables in amyotrophic lateral sclerosis (ALS): a neuroimaging study of ALS motor phenotype heterogeneity and cortical focality. J Neurol Neurosurg Psychiatry. 2013;84:766-73. Analysis of the motor homunclus topography using structual MRI changes in relation to clinical features in ALS.

54. Menke RA, Korner S, Filippini N, Douaud G, Knight S, Talbot K, et al. Widespread grey matter pathology dominates the longitudinal cerebral MRI and clinical landscape of amyotrophic lateral sclerosis. Brain J Neurol. 2014;137:2546-55. A large longitudinal MRI study in ALS, revealing extensive white matter damage cross-sectionally, and widespread greay matter involvement with time.

55. Lillo P, Mioshi E, Burrell JR, Kiernan MC, Hodges JR, Hornberger M. Grey and white matter changes across the amyotrophic lateral sclerosis-frontotemporal dementia continuum. PLoS One. 2012;7:e43993.

56. Rajagopalan V, Pioro EP. Distinct patterns of cortical atrophy in ALS patients with or without dementia: an MRI VBM study. Amyotroph Lateral Scler Frontotemporal Degen. 2014;15:216-25.

57. Verstraete E, Veldink JH, Hendrikse J, Schelhaas HJ, van den Heuvel MP, van den Berg LH. Structural MRI reveals cortical thinning in amyotrophic lateral sclerosis. J Neurol Neurosurg Psychiatry. 2012;83:383-8.

58. Schuster C, Kasper E, Machts J, Bittner D, Kaufmann J, Benecke $\mathrm{R}$, et al. Focal thinning of the motor cortex mirrors clinical features of amyotrophic lateral sclerosis and their phenotypes: a neuroimaging study. J Neurol. 2013;260:2856-64.

59. Walhout R, Westeneng HJ, Verstraete E, Hendrikse J, Veldink JH, van den Heuvel MP, et al. Cortical thickness in ALS: towards a marker for upper motor neuron involvement. J Neurol Neurosurg Psychiatry. 2015;86:288-94. First application of cortical thickness measurement to patients from the MND spectrum, uniquely also involving a disease control group, demonstrating specificity of cortical thinning for clinical UMN involvement.

60. Schuster C, Kasper E, Dyrba M, Machts J, Bittner D, Kaufmann J, et al. Cortical thinning and its relation to cognition in amyotrophic lateral sclerosis. Neurobiol Aging. 2014;35:240-6.

61. Geser F, Brandmeir NJ, Kwong LK, Martinez-Lage M, Elman L, McCluskey L, et al. Evidence of multisystem disorder in wholebrain map of pathological TDP-43 in amyotrophic lateral sclerosis. Arch Neurol. 2008;65:636-41.

62. Swinnen B, Robberecht $\mathrm{W}$. The phenotypic variability of amyotrophic lateral sclerosis. Nat Rev Neurol. 2014;10:661-70.

63. Bede P, Elamin M, Byrne S, McLaughlin RL, Kenna K, Vajda A, et al. Basal ganglia involvement in amyotrophic lateral sclerosis. Neurology. 2013;81:2107-15.

64. Westeneng HJ, Verstraete E, Walhout R, Schmidt R, Hendrikse J, Veldink JH, et al. Subcortical structures in amyotrophic lateral sclerosis. Neurobiol Aging. 2015;36:1075-82.

65. Takeda T, Uchihara T, Arai N, Mizutani T, Iwata M. Progression of hippocampal degeneration in amyotrophic lateral sclerosis with or without memory impairment: distinction from Alzheimer disease. Acta Neuropathol. 2009;117:35-44.

66. Prell T, Grosskreutz J. The involvement of the cerebellum in amyotrophic lateral sclerosis. Amyotroph Lateral Scler Frontotemporal Degen. 2013;14:507-15.

67. Tan RH, Devenney E, Dobson-Stone C, Kwok JB, Hodges JR, Kiernan MC, et al. Cerebellar integrity in the amyotrophic lateral sclerosis-frontotemporal dementia continuum. PLoS One. 2014;9: e105632.

68. King A, Maekawa S, Bodi I, Troakes C, Al-Sarraj S. Ubiquitinated, p62 immunopositive cerebellar cortical neuronal inclusions are evident across the spectrum of TDP-43 proteinopathies but are only rarely additionally immunopositive for phosphorylation-dependent TDP-43. Neuropathology. 2011;31:239-49.

69. Ciccarelli O, Catani M, Johansen-Berg H, Clark C, Thompson A. Diffusion-based tractography in neurological disorders: concepts, applications, and future developments. Lancet Neurol. 2008;7: 715-27.

70. Blain CR, Brunton S, Williams VC, Leemans A, Turner MR, Andersen PM, et al. Differential corticospinal tract degeneration in homozygous 'D90A' SOD-1 ALS and sporadic ALS. J Neurol Neurosurg Psychiatry. 2011;82:843-9. 
71. Verstraete E, Polders DL, Mandl RC, Van Den Heuvel MP, Veldink JH, Luijten P, et al. Multimodal tract-based analysis in ALS patients at 7T: a specific white matter profile? Amyotroph Lateral Scler Frontotemporal Degen. 2014;15:84-92.

72. Brettschneider J, Toledo JB, Van Deerlin VM, Elman L, McCluskey L, Lee VM, et al. Microglial activation correlates with disease progression and upper motor neuron clinical symptoms in amyotrophic lateral sclerosis. PLoS One. 2012;7:e39216.

73. Miller KL, Stagg CJ, Douaud G, Jbabdi S, Smith SM, Behrens TE, et al. Diffusion imaging of whole, post-mortem human brains on a clinical MRI scanner. NeuroImage. 2011;57:167-81.

74. Kwan JY, Jeong SY, Van Gelderen P, Deng HX, Quezado MM, Danielian LE, et al. Iron accumulation in deep cortical layers accounts for MRI signal abnormalities in ALS: correlating 7 tesla MRI and pathology. PLoS One. 2012;7:e35241.

75. Foerster BR, Dwamena BA, Petrou M, Carlos RC, Callaghan BC, Pomper MG. Diagnostic accuracy using diffusion tensor imaging in the diagnosis of ALS: a meta-analysis. Acad Radiol. 2012;19: 1075-86.

76. Ince PG, Evans J, Knopp M, Forster G, Hamdalla HH, Wharton $\mathrm{SB}$, et al. Corticospinal tract degeneration in the progressive muscular atrophy variant of ALS. Neurology. 2003;60:1252-8.

77. Senda J, Kato S, Kaga T, Ito M, Atsuta N, Nakamura T, et al. Progressive and widespread brain damage in ALS: MRI voxelbased morphometry and diffusion tensor imaging study. Amyotroph Lateral Scler Off Publ World Fed Neurol Res Group Motor Neuron Dis. 2011;12:59-69.

78. Trojsi F, Corbo D, Caiazzo G, Piccirillo G, Monsurro MR, Cirillo $\mathrm{S}$, et al. Motor and extramotor neurodegeneration in amyotrophic lateral sclerosis: a $3 \mathrm{~T}$ high angular resolution diffusion imaging (HARDI) study. Amyotroph Lateral Scler Frontotemporal Degen. 2013;14:553-61.

79. Verstraete E, Veldink JH, Mandl RC, van den Berg LH, van den Heuvel MP. Impaired structural motor connectome in amyotrophic lateral sclerosis. PLoS One. 2011;6:e24239.

80. Buchanan CR, Pettit LD, Storkey AJ, Abrahams S, Bastin ME. Reduced structural connectivity within a prefrontal-motorsubcortical network in amyotrophic lateral sclerosis. J Magn Reson Imaging. 2014.

81. Al-Sarraj S, King A, Troakes C, Smith B, Maekawa S, Bodi I, et al. p62 positive, TDP-43 negative, neuronal cytoplasmic and intranuclear inclusions in the cerebellum and hippocampus define the pathology of C9orf72-linked FTLD and MND/ALS. Acta Neuropathol. 2011;122:691-702.

82. Andersen PM, Al-Chalabi A. Clinical genetics of amyotrophic lateral sclerosis: what do we really know? Nat Rev Neurol. 2011;7:603-15.

83. Chio A, Mora G, Sabatelli M, Caponnetto C, Traynor BJ, Johnson $\mathrm{JO}$, et al. CHCH10 mutations in an Italian cohort of familial and sporadic amyotrophic lateral sclerosis patients. Neurobiol Aging. 2015;36:1767. e3-6.

84. Kassubek J, Muller HP, Del Tredici K, Brettschneider J, Pinkhardt $\mathrm{EH}$, Lule D, et al. Diffusion tensor imaging analysis of sequential spreading of disease in amyotrophic lateral sclerosis confirms patterns of TDP-43 pathology. Brain J Neurol. 2014;137:1733-40. An innovative attempt to explore in vivo white matter tract involvement in ALS patients in relation to the post mortem histopathological stages previously demonstrated by members of the same group, showing indications of conformity.

85. Roche JC, Rojas-Garcia R, Scott KM, Scotton W, Ellis CE, Burman R, et al. A proposed staging system for amyotrophic lateral sclerosis. Brain J Neurol. 2012;135:847-52.

86. Smith SM, Fox PT, Miller KL, Glahn DC, Fox PM, Mackay CE, et al. Correspondence of the brain's functional architecture during activation and rest. Proc Natl Acad Sci U S A. 2009;106:13040-5.
87. Schoenfeld MA, Tempelmann C, Gaul C, Kuhnel GR, Duzel E, Hopf JM, et al. Functional motor compensation in amyotrophic lateral sclerosis. J Neurol. 2005;252:944-52.

88. Mohammadi B, Kollewe K, Samii A, Dengler R, Munte TF. Functional neuroimaging at different disease stages reveals distinct phases of neuroplastic changes in amyotrophic lateral sclerosis. Hum Brain Mapp. 2011;32:750-8.

89. Witiuk K, Fernandez-Ruiz J, McKee R, Alahyane N, Coe BC, Melanson $\mathrm{M}$, et al. Cognitive deterioration and functional compensation in ALS measured with fMRI using an inhibitory task. J Neurosci Off J Soc Neurosci. 2014;34:14260-71.

90. Raaphorst J, van Tol MJ, Groot PF, Altena E, van der Werf YD, Majoie CB, et al. Prefrontal involvement related to cognitive impairment in progressive muscular atrophy. Neurology. 2014;83: 818-25.

91. Lowe MJ, Dzemidzic M, Lurito JT, Mathews VP, Phillips MD. Correlations in low-frequency BOLD fluctuations reflect corticocortical connections. NeuroImage. 2000;12:582-7.

92. Sporns O, Zwi JD. The small world of the cerebral cortex. Neuroinformatics. 2004;2:145-62.

93. Mohammadi B, Kollewe K, Samii A, Krampfl K, Dengler R, Munte TF. Changes of resting state brain networks in amyotrophic lateral sclerosis. Exp Neurol. 2009;217:147-53.

94. Greicius MD, Krasnow B, Reiss AL, Menon V. Functional connectivity in the resting brain: a network analysis of the default mode hypothesis. Proc Natl Acad Sci U S A. 2003;100:253-8.

95. van den Heuvel MP, Mandl RC, Kahn RS, Hulshoff Pol HE. Functionally linked resting-state networks reflect the underlying structural connectivity architecture of the human brain. Hum Brain Mapp. 2009;30:3127-41.

96. De Luca M, Smith S, De Stefano N, Federico A, Matthews PM. Blood oxygenation level dependent contrast resting state networks are relevant to functional activity in the neocortical sensorimotor system. Exp Brain Res. 2005;167:587-94.

97. Lowe MJ, Phillips MD, Lurito JT, Mattson D, Dzemidzic M, Mathews VP. Multiple sclerosis: low-frequency temporal blood oxygen level-dependent fluctuations indicate reduced functional connectivity initial results. Radiology. 2002;224:184-92.

98. Jelsone-Swain LM, Fling BW, Seidler RD, Hovatter R, Gruis K, Welsh RC. Reduced interhemispheric functional connectivity in the motor cortex during rest in limb-onset amyotrophic lateral sclerosis. Front Syst Neurosci. 2010;4:158.

99. Tedeschi G, Trojsi F, Tessitore A, Corbo D, Sagnelli A, Paccone $\mathrm{A}$, et al. Interaction between aging and neurodegeneration in amyotrophic lateral sclerosis. Neurobiol Aging. 2012;33:886-98.

100. Douaud G, Filippini N, Knight S, Talbot K, Turner MR. Integration of structural and functional magnetic resonance imaging in amyotrophic lateral sclerosis. Brain J Neurol. 2011;134: 3470-9.

101. Agosta F, Valsasina P, Absinta M, Riva N, Sala S, Prelle A, et al. Sensorimotor functional connectivity changes in amyotrophic lateral sclerosis. Cereb Cortex (New York 1991). 2011;21:2291-8.

102. Zhou F, Xu R, Dowd E, Zang Y, Gong H, Wang Z. Alterations in regional functional coherence within the sensory-motor network in amyotrophic lateral sclerosis. Neurosci Lett. 2014;558:192-6.

103. Agosta F, Canu E, Valsasina P, Riva N, Prelle A, Comi G, et al. Divergent brain network connectivity in amyotrophic lateral sclerosis. Neurobiol Aging. 2013;34:419-27.

104. Schmidt R, Verstraete E, de Reus MA, Veldink JH, van den Berg LH, van den Heuvel MP. Correlation between structural and functional connectivity impairment in amyotrophic lateral sclerosis. Hum Brain Mapp. 2014;35:4386-95.

105. Trojsi F, Esposito F, de Stefano M, Buonanno D, Conforti FL, Corbo D, et al. Functional overlap and divergence between ALS and bvFTD. Neurobiol Aging. 2015;36:413-23. 
106. Proudfoot M, Woolrich MW, Nobre AC, Turner MR. Magnetoencephalography. Pract Neurol. 2014;14:336-43.

107. Pioro EP, Antel JP, Cashman NR, Arnold DL. Detection of cortical neuron loss in motor neuron disease by proton magnetic resonance spectroscopic imaging in vivo. Neurology. 1994;44:1933-8.

108. Stagg CJ, Knight S, Talbot K, Jenkinson M, Maudsley AA, Turner MR. Whole-brain magnetic resonance spectroscopic imaging measures are related to disability in ALS. Neurology. 2013;80: $610-5$.

109. Pioro EP, Majors AW, Mitsumoto H, Nelson DR, Ng TC. 1HMRS evidence of neurodegeneration and excess glutamate + glutamine in ALS medulla. Neurology. 1999;53:71-9.

110. Foerster BR, Callaghan BC, Petrou M, Edden RA, Chenevert TL, Feldman EL. Decreased motor cortex gamma-aminobutyric acid in amyotrophic lateral sclerosis. Neurology. 2012;78:1596-600.

111. Bede P, Bokde AL, Byrne S, Elamin M, Fagan AJ, Hardiman O. Spinal cord markers in ALS: diagnostic and biomarker considerations. Amyotroph Lateral Scler Off Publ World Fed Neurol Res Group Motor Neuron Dis. 2012;13:407-15.

112. Cohen-Adad J, El Mendili MM, Morizot-Koutlidis R, Lehericy S, Meininger V, Blancho S, et al. Involvement of spinal sensory pathway in ALS and specificity of cord atrophy to lower motor neuron degeneration. Amyotroph Lateral Scler Frontotemporal Degen. 2012;14:30-8. Important early demonstration of the great potential of advanced spinal cord MRI in furthering understanding of the evolution of in vivo pathology in ALS.

113. Al-Chalabi A, Jones A, Troakes C, King A, Al-Sarraj S, van den Berg LH. The genetics and neuropathology of amyotrophic lateral sclerosis. Acta Neuropathol. 2012;124:339-52.

114. Mahoney CJ, Beck J, Rohrer JD, Lashley T, Mok K, Shakespeare $\mathrm{T}$, et al. Frontotemporal dementia with the C9ORF72 hexanucleotide repeat expansion: clinical, neuroanatomical and neuropathological features. Brain J Neurol. 2012;135:736-50.

115. Bede P, Bokde AL, Byrne S, Elamin M, McLaughlin RL, Kenna $\mathrm{K}$, et al. Multiparametric MRI study of ALS stratified for the C9orf72 genotype. Neurology. 2013;81:361-9.

116. Cistaro A, Pagani M, Montuschi A, Calvo A, Moglia C, Canosa A, et al. The metabolic signature of C9ORF72-related ALS: FDG PET comparison with nonmutated patients. Eur J Nucl Med Mol Imaging. 2014;41:844-52.

117. Benatar M, Wuu J. Presymptomatic studies in ALS: rationale, challenges, and approach. Neurology. 2012;79:1732-9.

118. Ng MC, Ho JT, Ho SL, Lee R, Li G, Cheng TS, et al. Abnormal diffusion tensor in nonsymptomatic familial amyotrophic lateral sclerosis with a causative superoxide dismutase 1 mutation. J Magn Reson Imaging. 2008;27:8-13.

119. Vucic S, Winhammar JM, Rowe DB, Kiernan MC. Corticomotoneuronal function in asymptomatic SOD-1 mutation carriers. Clin Neurophysiol. 2010;121:1781-5.

120. Carew JD, Nair G, Andersen PM, Wuu J, Gronka S, Hu X, et al. Presymptomatic spinal cord neurometabolic findings in SOD1positive people at risk for familial ALS. Neurology. 2011;77: 1370-5.

121. Dopper EG, Rombouts SA, Jiskoot LC, Heijer TD, Graaf JR, Koning ID, et al. Structural and functional brain connectivity in presymptomatic familial frontotemporal dementia. Neurology. 2013;80:814-23.

122. Evans MC, Serres S, Khrapitchev AA, Stolp HB, Anthony DC, Talbot K, et al. T(2)-weighted MRI detects presymptomatic pathology in the SOD1 mouse model of ALS. J Cereb Blood Flow Metab Off J Int Soc Cereb Blood Flow Metab. 2014;34:785-93. Demonstration of the applicability of advanced MRI to mouse models of ALS, with insights into the neuroinflammatory landscape prior to motor symptoms through a combined histological analysis.

123. Evans MC, Gaillard PJ, de Boer M, Appeldoorn C, Dorland R, Sibson NR, et al. CNS-targeted glucocorticoid reduces pathology in mouse model of amyotrophic lateral sclerosis. Acta Neuropathol Commun. 2014;2:66.

124. Foerster BR, Carlos RC, Dwamena BA, Callaghan BC, Petrou M, Edden RAE, et al. Multimodal MRI as a diagnostic biomarker for amyotrophic lateral sclerosis. Ann Clin Transl Neurol. 2014;1: 107-14.

125. Cervo A, Cocozza S, Sacca F, Giorgio SM, Morra VB, Tedeschi $\mathrm{E}$, et al. The combined use of conventional MRI and MR spectroscopic imaging increases the diagnostic accuracy in amyotrophic lateral sclerosis. Eur J Radiol. 2015;84:151-7.

126. Menke RAL, Gray E, Lu CH, Kuhle J, Talbot K, Malaspina A, et al. CSF neurofilament light chain reflects corticospinal tract degeneration in ALS. Ann Clin Transl Neurol. 2015; in press.

127. Turner MR, Grosskreutz J, Kassubek J, Abrahams S, Agosta F, Benatar M, et al. Towards a neuroimaging biomarker for amyotrophic lateral sclerosis. Lancet Neurol. 2011;10:400-3.

128. Wood H. Alzheimer disease: [(11)C]PBB3-a new PET ligand that identifies tau pathology in the brains of patients with AD. Nat Rev Neurol. 2013;9:599. 